Introduction Syphilis and Human immunodeficiency virus (HIV) infections have been found to be major public health problems in Sub-Saharan Africa. Clinical presentation of syphilis in patients infected with HIV has been described as atypical. This study was aimed to determining the seroprevalence and risk factors of syphilis among HIV infected patients in a tertiary hospital in Nigeria.

Methods It is a descriptive cross-sectional survey of 793 HIVinfected patients enrolled at the Anti-Retroviral Treatment (ART) clinic, University College Hospital, Ibadan, Nigeria between July and December 2010. Detailed medical history was obtained from the patients after informed consent. Screening for syphilis using qualitative rapid Plasma Reagin (RPR) was performed on each of the sera/plasma collected from them. Measurement of CD4 + T lymphocyte (CD4) count was carried out by flow cytometry and Roche Ampiclor RNA PCR assay was used for the measurement of plasma HIV RNA (viral load). Data analysis was performed using SPSS version 23.

Results A total of 793 participants, 557 females and 236 males were enrolled. The mean age of the patients was $36.99 \pm 10.3$ years. The overall prevalence of syphilis among this cohort of patients was $1.51 \%$ (1.4\% in females and $1.7 \%$ in males). $30-39$ years age groups were mostly affected with $50.0 \%$ of the cases of syphilis. The mean age, weight, CD4 count and log viral load for the syphilis co-infected HIV patients were $38.17 \pm 8.22$ years, 59.8 $\pm 10.5 \mathrm{Kg}, 275.92 \pm 282.1 \mathrm{cells} / \mathrm{m}^{\mathrm{m} 3}$ and $4.35 \pm 1.37 \mathrm{copies} / \mathrm{ml}$ respectively compared to $36.97 \pm 10.3$ years, $56.7 \pm 15.5 \mathrm{~kg}$, $262.06 \pm 256.5 \mathrm{cells} / \mathrm{mm}^{3}$ and $4.62 \pm 1.2$ copies $/ \mathrm{ml}$ respectively in the population without syphilis co-infection. Syphilis co-infection occurs more among females (OR 1.1, 95\% CI (0.75-1.58), and the married (OR 1.1, 95\% CI (0.75-1.58) without significant association.

Conclusion Our study had revealed a low prevalence of syphilis in patients living with HIV/AIDS. Routine screening and counselling for syphilis should be considered for patients in AIDS care in Sub-Saharan Africa.

\section{P3.56 RISK FACTORS ASSOCIATED WITH SEXUALLY TRANSMITTED INFECTIONS AMONG HIV-INFECTED PREGNANT WOMEN IN SOUTH AFRICA}

Dvora Joseph Davey ${ }^{1,2},{ }^{3} \mathrm{~A}$ Andrew Medina-Marino, ${ }^{3} \mathrm{M}$ Mudau, ${ }^{3}$ Lindsey De Vos, ${ }^{3}$ Dawie Olivier, ${ }^{4,5}$ Remco P Peters, ${ }^{4}$ James A McIntyre, ${ }^{2}$ Jeffrey D Klausner. ${ }^{1}$ Faculty of Health Sciences, University of Cape Town, Cape Town, South Africa; ${ }^{2}$ David Geffen School of Medicine, UCLA, Los Angeles, CA; ${ }^{3}$ Research Unit, Foundation for Professional Development, Pretoria, South Africa; ${ }^{4}$ Anova Health Institute, Johannesburg, South Africa; ${ }^{5}$ Department of Medical Microbiology, Faculty of Health Sciences, University of Pretoria, Pretoria, South Africa

\subsection{6/sextrans-2017-053264.291}

Introduction Sexually transmitted infections (STI) may increase the risk of perinatal HIV transmission; however, there is limited evidence on risk factors associated with STIs among HIVinfected pregnant women.

Methods We conducted a study of HIV-infected pregnant women $(n=199)$ in antenatal care at 2 primary care facilities in South Africa to evaluate risk factors for STIs. Participants were interviewed and self-collected vulvovaginal swabs which were tested for Chlamydia trachomatis (CT), Neisseria gonorrhoea (NG) and Trichomonas vaginalis (TV), Xpert (Cepheid, Sunnyvale, USA). We report descriptive and multivariate logistic regression results of factors associated with any STI.
Results Median age was 29 years; median gestational age was 20 weeks. Prevalence of any STI was 51\%. Of all women $90 \%$ reported having sex during pregnancy (of which 9\% reported oral sex and $4 \%$ anal sex). Most (62\%) had sex 7 days prior to study enrolment. Over $70 \%$ of women were not virally suppressed (>200 copies $/ \mathrm{mL}$ ) while $99 \%$ of women were on antiretroviral therapy. At last sex $75 \%$ reported condomless sex and $15 \%$ reported having $>1$ sex partner in the past 12 months. Of women $14 \%$ reported any alcohol use during pregnancy of whom $25 \%$ reported $\geq 5$ drinks on a typical day. Twenty-two percent reported being in a serodiscordant relationship with father of the child; $27 \%$ seroconcordant relationship; 51\% didn't know. Odds of having any STI decreased as age increased $(\mathrm{OR} /$ year $=0.93 ; 95 \% \mathrm{CI}=0.88-$ 0.98). Odds increased with increased gestational age at time of testing $(\mathrm{aOR}=1.07 ; 95 \% \mathrm{CI}=1.02-1.12)$ and recent sex (past 30 days vs. longer $)(\mathrm{aOR}=1.42 ; 95 \% \mathrm{CI}=1.04-1.93)$. Trends toward increased odds of any STI included: $>1$ partner $(\mathrm{aOR}=2.36 ; 95 \% \mathrm{CI}=0.96-5.84 ; \mathrm{p}=0.06)$ and $\geq 5$ drinks daily $(\mathrm{aOR}=3.61 ; 95 \% \mathrm{CI}=0.77-35.7 ; \mathrm{p}=0.09)$, adjusted for age.

Conclusion Nearly all HIV-infected pregnant women were sexually active during pregnancy with behaviours associated with increased risk for STIs: condomless sex, anal sex, alcohol use or multiple partners. Interventions to reduce STI risk during pregnancy are urgently needed.

\section{P3.57 KNOWLEDGE AND ATTITUDE OF DENTAL STUDENTS TOWARDS THE DENTAL TREATMENT OF PATIENTS WITH HIV/AIDS IN MANGALORE CITY}

Eby Aluckal. 1 Mar Baselios Dental College, Kothamangalam, Ernakulam, India

\subsection{6/sextrans-2017-053264.292}

Introduction Oral health care of patients with human immunodeficiency virus (HIV)/acquired immune-deficiency syndrome (AIDS) is a growing area of concern. Information on HIV and AIDS related knowledge among dental students provides a crucial foundation for efforts aimed at developing an appropriate dental curriculum on HIV and AIDS. The purpose of this study was to assess the knowledge and attitude of Indian clinical dental students towards the treatment of patients with HIV/AIDS and perceived sources of information regarding HIV-related issues.

Methods Data were collected from clinical dental students (third year, fourth year and internship) from four dental colleges in Mangalore city. The questions assessed the knowledge and attitude towards treatment of patients with HIV and the perceived source of information related to HIV/AIDS.

Results The willingness to treat HIV-positive patients among dental students was $67.0 \%$, and $74.20 \%$ were confident of treating a patient with HIV/AIDS. The potential problems in rendering treatment to these patients were effect on the attitude of other patients (49.90\%) and staff fears (52.50\%). The correct knowledge regarding the infection-control practice (barrier technique) was found among only $15.50 \%$ of respondents. The respondents had sufficient knowledge regarding the oral manifestations of HIV/AIDS.

Conclusions No correlation was found between the knowledge and attitude score, demonstrating a gap between knowledge and attitude among the dental students regarding treatment of HIV-infected patients. Appropriate information has to be delivered through the dental education curriculum, which can instil confidence in students about their ability to manage HIV-positive patients. 